\title{
An Intelligent Healthcare Serviceto Monitor Vital Signs in Daily Life - A Case Study on Health-IoT
}

\author{
Mobyen Uddin Ahmed \\ School of Innovation, Design and Engineering, Mälardalen University, Sweden
}

\begin{abstract}
Vital signs monitoring for elderly in daily life environment is a promising concept that efficiently can provide medical services to people at home. However, make the system self-served and functioning as personalized provision makes the challenge even larger. This paper presents a case study on a Health-IoT system where an intelligent healthcare service is developed to monitor vital signs in daily life. Here, a generic Health-IoT framework with a Clinical Decision Support System (CDSS) is presented. The generic framework is mainly focused on the supporting sensors, communication media, secure and safe data communication, cloud-based storage, and remote accesses of the data. The CDSS is used to provide a personalized report on persons' health condition based on daily basis observation on vital signs. Six participants, from Spain $(n=3)$ and Slovenia $(n=3)$ have been using the proposed healthcare system for eight weeks (e.g. 300+ health measurements) in their home environments to monitor their health. The sensitivity, specificity and overall accuracy of the DSS's classification are achieved as $90 \%, 97 \%$ and $96 \%$ respectively while $\mathrm{k}=2$ i.e., top 2 most similar retrieved cases are considered. The initial user evaluation resultdemonstrates the feasibility and performance of the implemented system through the proposed framework.
\end{abstract}

\section{INTRODUCTION}

Self-served e-health systems for health monitoring in the home is an area of increasing interest. According to WHO, healthy ageing is vital for countries' economic development since it is one of the three pillars of active ageing. Around two billion people i.e., one out of every four will be older than 60 years in the year 2050. Again, worldwide demographics show increasing numbers of elderly people, and in most European countries this is combined with a lower number of younger people. This means that the cost of caring for elderly is increasing for the taxpayers. Hence, new and cost-effective ways to provide health services is a prerequisite if we should be able to keep the current levels of health service in the future. Healthcare at home is appealing for both primary users and caregivers. Users can remain in their home environment as patients, and the caregivers will have a reduced cost for housing and patient beds. Care at home when given by physically travelling caregivers can however mean that a lot of work time is wasted when caregiving staff is travelling between the homes of patients. E-health systems can reduce the physical travel of caregiver staff, replacing travel with virtual visits in the homes through computerized support systems [9][5].Thus, the health monitoring system for elderly is not limited to take place in primary care facilities simply due to deployment of ICT as addressed in the reviews [9],[10],[11].

In order for e-health services to be a potential replacement of physical visits by patients to caregiver or by caregiver to patient, e-health services must be reliable and safe. A patient being monitored at home by an e-health system must be as safe as a patient being monitored at a hospital. Internet of Things is maturing and the emerging standards are useful for e-health services [9][1]. Many current projects are considering the issue; such as the H@H system [7] gathers the wearable sensors data, which continuously monitor patients' physio-pathological cardiovascular and respiratory parameters. Similarly, authors in [8], presented an intelligent home-based platform, i.e. the iHome Health-IoT, where a framework is implemented through IoT. Today, sensor data are traveling from sensors to a remote server, data is analysed remotely in a distributed manner, and health status of a user is presented in real-time. However, to support several of these facilities in sensor data management an efficient framework is necessary and beneficial. Research also shows that several projects like eCAALYX and HELP are ongoing which provide home healthcare for elderly [24]. Similarly, due to the need of ICT and globalization, several web sites or online-based healthcare service providers are available in the market, which provide possibilities to monitor health parameters such as blood pressure, blood glucose [12][13], and activity [14]. However, most of them provide feedback, recommendation, alarm messages based on a set of general rules for an individual healthrelated parameter [4][5]. 
This paper presents a case study on an intelligent Health-IoT framework to monitor health in daily life. Here, the proposed framework combines general rules with a case-based reasoning (CBR) approach [15], [16] to provided personalized self-served health-monitoring system for elderly in home environment. A set of general rules has used to classify individual health parameters such as 'blood pressure is high' is an instance/value for the health parameter blood pressure. However, the message is not personal as well as not provides overall status of user's health condition. A case-based reasoning (CBR) [17],[19] approach can work in a way close to human reasoning e.g. solves a new problem applying previous experiences, which is more common for doctors, clinicians or engineers. Aamodt and Plaza has introduced a life cycle of CBR [17] with four main steps Retrieve, Reuse, Revise and Retain present key tasks to implement such kind of cognitive model.The advantages of CBR in the medical domain have identified in several research works i.e. in [17], [18], [19], [20] and [27]. The proposed approach applies CBR to combine several different health parameters, user's contextual, medical, medicine related information, which generates an overall classification of health condition such as 'current health condition is healthy'. In addition, the system generates feedback, recommendation and alarm in a personalized manner based on elderly's medical information and health parameters such as blood pressure, blood glucose, weight, activity, pulse, etc. A light version of the Health-IoT framework for health monitoring system is implemented and evaluated in the scope of the SAAPHO (Secure Active Aging: Participation and Health for the Old) project. Six participants from Spain $(n=3)$ and Slovenia $(n=3)$ have used the system for 8 weeks and the evaluation is conducted in threecategories: 1) proof-of-concept prototype 2) classification accuracy of the CBR retrieval has observed where percentages of correctly classified cases, sensitivity, specificity and overall accuracy have calculated, and 3) a user evaluation. The initial evaluation result demonstrates the feasibility and performance of the implemented system through the proposed framework. Again, the acceptance level and usefulness of the healthcare system was found very high by most of the users. Moreover, the feedbacks recommendations and alarm messages generated by the proposed system are personalized and differ from the general messages.

\section{A CASE STUDY ON HEALTH-IOT}

The aim of a self-serve health monitoring system is to enable to follow the health trends of elderlyin home environment, and to detect health deterioration at an early stage. The system should also provide real-time feedback, and enable historical information, reminder and recommendations. Here, sensor unit contains several sensors powered by battery; unit for elderly is a Tablet contains collector with a user interface programmed in Android operating system; remote server stores the measurements considering safety, security, and privacy; finally, it generates feedbacks, reminders, recommendations, etc. Communication between sensors and collector is conducted using Bluetooth and communication between collector and remote server is connected by https \& SOAP communication. A rule-based classification method is applied to classify the measurements and generate real-time feedback; a blood pressure measurement 142/92 $\mathrm{mmHg}$ could be classifying as "High blood pressure" is one example. The goal of the historical information is to provide to the user a possibility to see and compare the summary in a graph for a specific range of dates. For example, considering medication compliance "total number of medication is taken", and "total number of medications are skipped" are presented. Reminders are generated which helps to monitor several health parameters regularly such as 'Devices_Not_Used' (if there is no measurement received over a week), 'Medications_skipped' (if a certain number of medications is skipped). Similarly, recommendations are generated such as 'Out_Of_Normal_Range' (if $70 \%$ of the measurements classes are outside the normal range considering a week), 'Fluctuation' (if the measurements over a week show fluctuation in more than 70\%), 'Weight_Loss' (if the current weight is less than $3 \mathrm{~kg}$ then weight received 3 months

ago),

'Activity_Increased/Activity_Decreased'

(considering one week measurements and a calculated slope value), etc. Moreover, a monthly summary is generated where maximum, minimum, average and standard deviation are considered.

\subsection{Overview of the Proposed Health-IoT Framework}

Remote health monitoring or e-health systems are developing increasingly day by day with the help of Internet of Things (IoT) [9][1][2]. Such systems are useful in several areas, from Ambient Assisted Living (AAL) environments for elderly [3][4][5] to healthy people for monitoring their health regularly i.e. athletes or sports men [6]. The aim of a self-serve health monitoring system is to send patient's (i.e. user's) health related information through sensor readings to a remote 
server (or cloud) where the remote server is accessible by clinicians, doctors, researchers, hospitals, patients etc. anywhere around the world as a characteristic of Internet of Things (IoT). An Expert System (ES) and/or a Decision Support System (DSS) will further analyse the sensor readings through the IoT and provide a real-time feedback to the patient/user immediately. A patient/user will have the possibility to access to see/compare the current health measurement with previous measurements and same time they can share the health record with their healthcare provider. An example of such self-serve health monitoring system is presented in Figure 1 as an overview of the system.

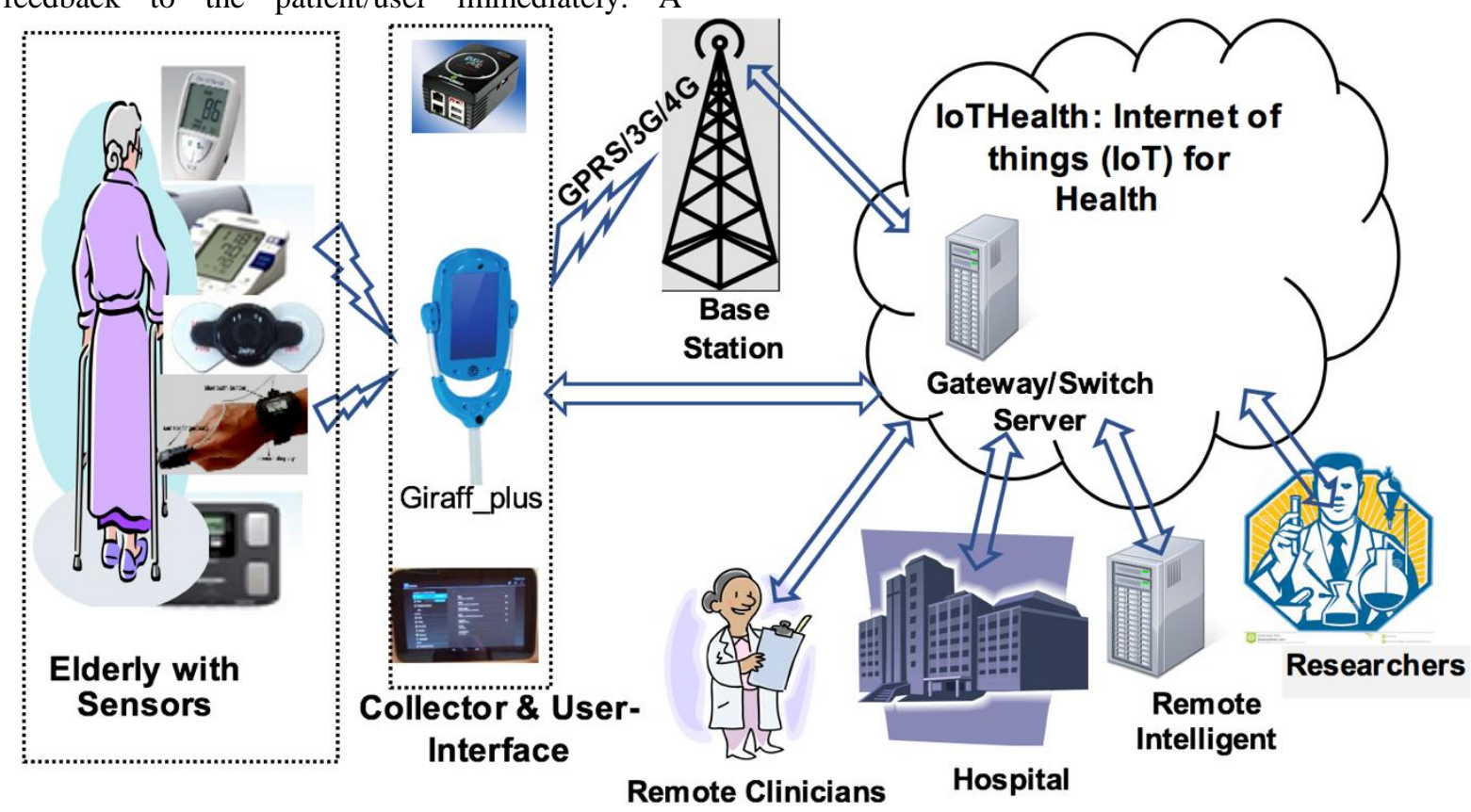

Figure 1. An Overview of the self-served health monitoring system through Internet of Things (IoT).

As can be seen form Figure 1, an elderly user uses several sensor devices to measure blood pressure, blood glucose, heart rate, pulse, weight etc. to monitor vital signs. Different commercial Bluetooth sensor devices were acquired to provide those parameters, for example, the blood pressure and weight monitoring devices are collected from a third-party vendor $\mathrm{OMRON}^{1}$; blood glucose is from BeneCheck ${ }^{2}$; hearth rate, respiration and activity from Zephyr ${ }^{3}$; and Pulse rate and oxygen saturation from WristOx $2^{4}$ etc. All the sensor readings are sent to the Internet of Things for Health (IoTHealth) via a collector. A collector is hardware like GuruPlug Server ${ }^{5}$ or an application running on a tablet or on Giraff_plus ${ }^{6}$, which convey the sensor readings to the cloud and collector sends the sensor reading using Wi-Fi as default. However, it is also possible that the sensor

${ }^{1}$ http://www.healthcare.omron.co.jp/bt/english/ ${ }^{2}$ http://www.glbiotech.com.tw/productdesc.php?pdtid=11 3 http://zephyranywhere.com/products/

${ }^{4}$ http://www.nonin.com/OEMSolutions/WristOx23150-OEM

5 https://www.globalscaletechnologies.com/tguruplugdetails.aspx

6http://www.giraffplus.eu/ readings can be sent via a base station using GPRS/3G/4G. In the tablet or Giraff_plus a userinterface is developed that present the real-time feedbacks on the measurements. Moreover, the user can see and compare previous measurements, which is plotted in a chart diagram. There is a remote intelligent health server where a Decision Support System (DSS) and an Expert System (ES) is running which analysis the sensor readings and provides recommendations and reminders to the user. The system also generates emergency alarms if it is necessary and sends them to clinicians/doctors. A generic system-level framework is proposed which enables different kinds of sensor devices, communications, storages, users, services, gateways, switches and etc

\subsection{Generic System Level Framework}

As can be seen from Figure 2, the proposed framework is divided mainly into four parts; 1) sensor unit, 2) collector and user-interface unit, 3) gateway and switch server and 4) intelligent health server. Beside them, there is another part in the framework named 'Third-party User-Interface' which aims is to connect existing software thathas 
been used by clinicians/doctors and healthcare providers/researchers. Here, users with proper permission can accesses patients' records via HTTPS web services to diagnosis, assist, research and other purposes. The communications between sensor and collector is open and can support different communication standards such as Bluetooth, Bluetooth low energy, IEEE 802.15.6 and ZigBee. The communication between collectors and cloud could be conducted both through web services (e.g. SOAP and/or RESTFul) as well as base station (e.g. GPRS/3G/4G). In the cloud, the servers i.e. gateway/switch server and intelligence health server could communicate using web services.

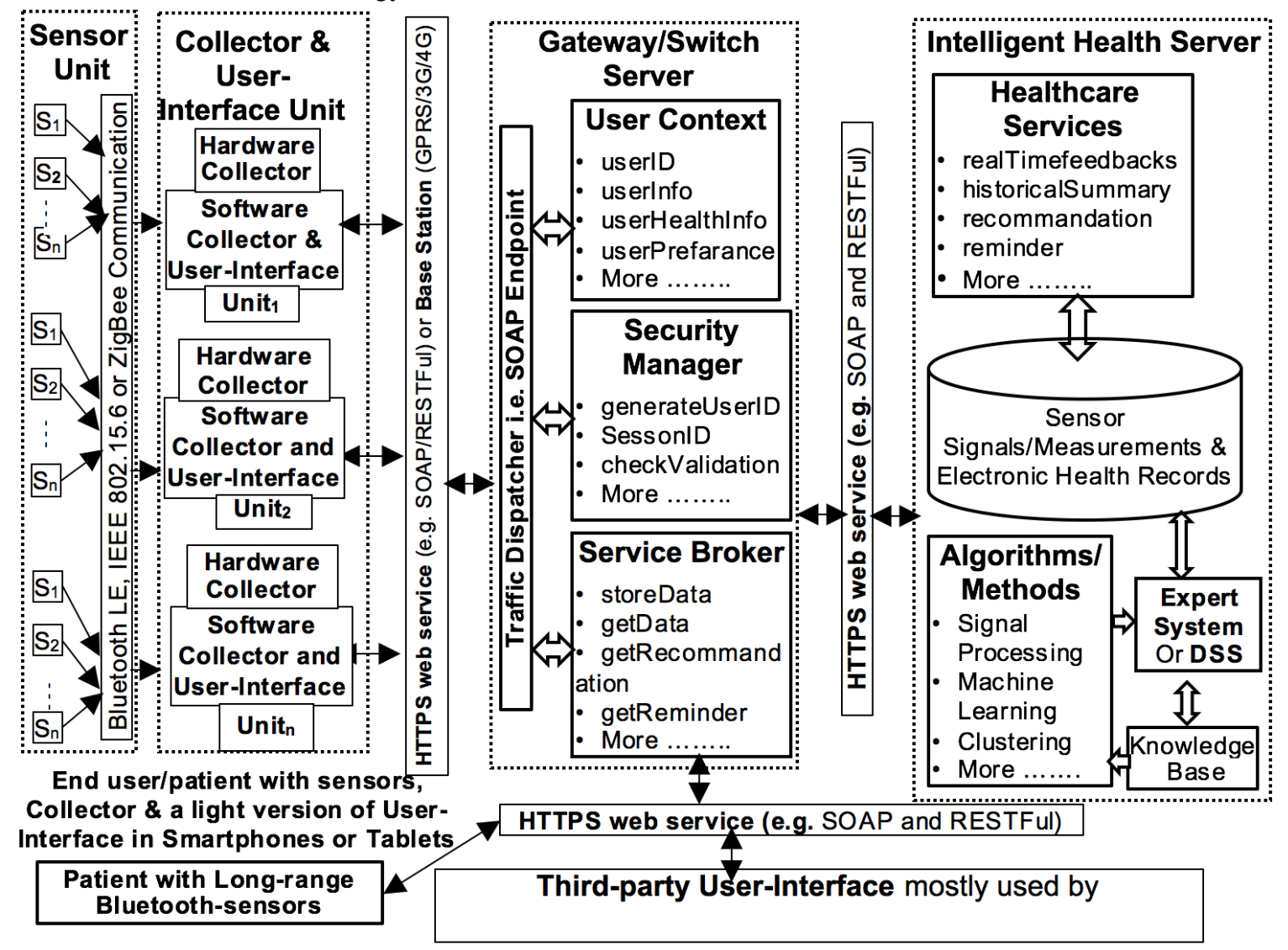

Figure 2. A generic system-level framework for self-served health monitoring system

Sensor unit: it contains several healthcare related sensor devices such as blood pressure, blood glucose, weight and etc. The sensor devices are run by battery and send their readings to the collector in a short distance communication such as Bluetooth or Zigbee. The sensors can send data continuously or discretely based on user health preference. The sensor devices are paired either with hardware like GuruPlug server or a Tablet where e.g. an Android operating system is installed into the collector units.

Collector and user-interface unit: this unit is combined with collector and a light version of userinterface. If it is a Tablet then collector is a software application, which accesses the Tablet's Bluetooth, Wi-Fi and 3G/4G for Communication. The collector can be a hardware device, which could also contain USB, Bluetooth, Wi-Fi and $3 \mathrm{G} / 4 \mathrm{G}$ for communication like a GuruPlug server.
A light version of the user-interface is developed that can be run on a user's smart phone or tablet. The user-interface can be developed in any common operating system, like Android, iOS, and Windows Phone. The main objective of the userinterface is to present real-time feedbacks; recommendations, reminders, alarms, historical summary etc. based on users health related measurements. Moreover, users can provide there contextual, medical, medicine and other information and same time they can set their health parameters and preferences.

Gateway and switch server: this a server, placed in the cloud, that work as a gateway and switch where Traffic Dispatcher (TD) is the main entry point and user send request via web services. TD takes the requests of the user such as login/logout, obtain adaptations user context, obtain messages of the different services connected to the system and 
obtain URLs of external services. When a user tries to login, theSecurity Manager (SM) is activated and it controls the access of the users and gateways to the system generating SessionIDs. To do that, it has aencrypted database with the information of the user, terminating the sending of information when a wrong behaviour, or an incorrect SessionID, is detected. When a new user wants to use the application, his/her data will be stored in the SM database. The user identifier used by the framework is the UUID (Universally Unique Identifier), which guarantees that the user is identified in a unique way by any component in the platform but also outside the framework. Each time that the user wants to use the application he/she will have to log into the User-Interface. This process involves the sending of the username and password to the SM and the generation of the sessionID if the data was right that is validation of sessionIDs and userIDs. TheServices Broker (SB) hosts a registry of descriptions of the services inside the framework but also of other services that could be useful for the users. For example, when a user is sending their measurements to store, or want to see previous stored information, they can face recommendations or reminders.

Intelligent health server: this is another server in the cloud, which mainly stores users' health related measurements both as raw and analysed data into the storage database. In the server, healthcare services are also implemented that generate realtime feedbacks, recommendations, reminders, historical summary, alarms, etc. Similarly, Expert Systems (ESs), Decision Support Systems (DSSs), knowledge base, a set of different algorithms and methods are also available to assist doctors/clinicians in better analysing, understanding and decision-making tasks.

\section{III.MATERIALS AND METHODS}

Applying a light version of the proposed framework through a project named SAAPHO, a prototype system has been developed where four sensor devices for healthcare are implemented and they are: 1) blood pressure, 2) blood glucose, 3) weight, and 4) activity. The sensor devices are run by battery and communicate via Bluetooth communication. The sensors are paired with an android tablet device where the User-Interface and the Collector are running. The sensor readings are transferred to the cloud via collector in the tablet using HTTPS SOAP web service. The system has implemented three different kinds of healthcare services: 1) real time feedback generation service, 2) historical summary calculation service, and 3) recommendation generation service [4][5].Data were collected from six participants from Spain $(n=3)$ and Slovenia $(n=3)$ have used the system for 8 weeks. They were invited to use the system at their own home for eight weeks (e.g. 2 months). The mean age of the 3 participants in Spain was 69.3 (SD: 9.9); 66-72 years and the mean age of the 3 participants in Slovenia was 65.7 (SD: 9.9); 6074 years. Regarding the gender both in Spain and Slovenia, $66.6 \%$ of the participants were women $(n=2)$ and $33.3 \%$ were men $(n=1) .100 \%$ of the participants had experience in using computers. $66.6 \%$ had experience in using tablet PC and all of them had Internet at home. The participants were asked to use the system at least once a day, which is, they should measure health related parameters once a day. After 8 weeks, 339 cases are collected, among them only 323 cases are selected to evaluate the proposed approach and rest of them are ignored due to missing values in the cases. The reason for missing values is due to bad connection of Bluetooth or Wi-Fi. An expert in the domain classifies the cases into three classes (e.g. 'Healthy', 'More or Less Healthy' and 'Need Further Diagnosis') and the evaluation results are presented in the result section.

\subsection{Personalized self-served health-monitoring system}

To promote a personalized self-served healthmonitoring the proposed approach combines general rules and a case-based reasoning (CBR) approach. The steps in process diagram of the proposed system for personalized health monitoring service are presented in Figure 2.

Step1 is used to collect sensor readings, there are different sensor devices are used to collect measurements for different vital signs of a user i.e., 1) Blood Pressure (BP), 2) Blood Glucose (BG), 3) Weight (WGT), 4) Activity (AC), 5) Heart Rate (HR) 6) Oxygen Saturation ( $\mathrm{SpO} 2)$ and etc. The sensor measurements are collected then transferred to step4 for feature extraction and a new case formulation. 


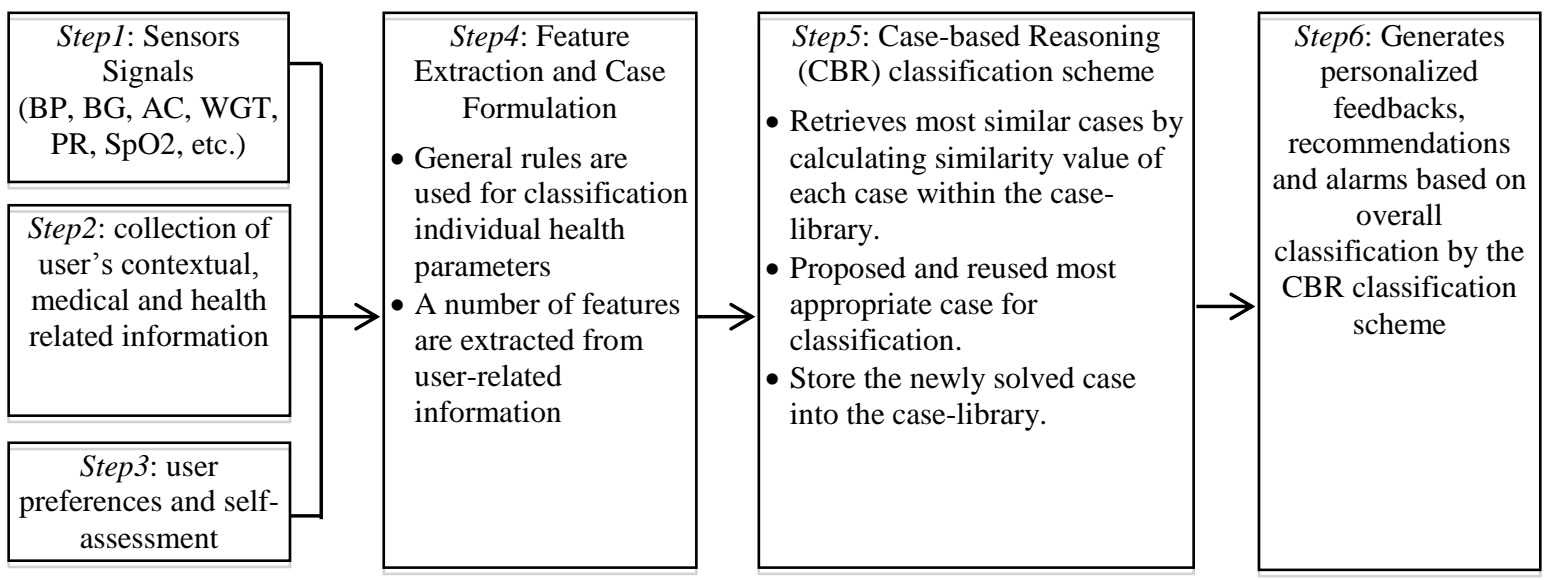

Figure 3. Steps diagram of the proposed system for personalized healthcare service.

In Step2, user contextual information such as height, age, gender, etc.; medical and health related information such as diseases, comorbidity is collected and transferred them to Step4for feature extraction.Similarly, Step3 collects user preferences on sensitivity of the vital signs and self-assessment about their health condition. To conclude the user's current health condition, the users were asked the following question:" How do you assess your overall health?" The userwas to choose one out of five options: 1. perfect, 2. good, 3. fair, 4. poor, 5. could not respond. All the information is then transferred to Step4 for feature
extraction.Step4 is used for feature extraction where a rule-based classification method is applied to classify the sensor signal measurements. For example, a blood pressure measurement 142/92 $\mathrm{mmHg}$ could be classified as "High" and/or BMI 26.1 by measuring user's weight could be classified "Overweight". A set of rules used in the classification method is mainly collected from the literature study [12] [13] [21] [22] [23], which is further validated through healthcare practitioners are presented in Table 1.

Table 1. Rules are used to generate real time feedback on each measurement.

\begin{tabular}{|c|c|}
\hline Health Parameters & Rules to Generate Real Time Feedback \\
\hline Blood Glucose (BG) & 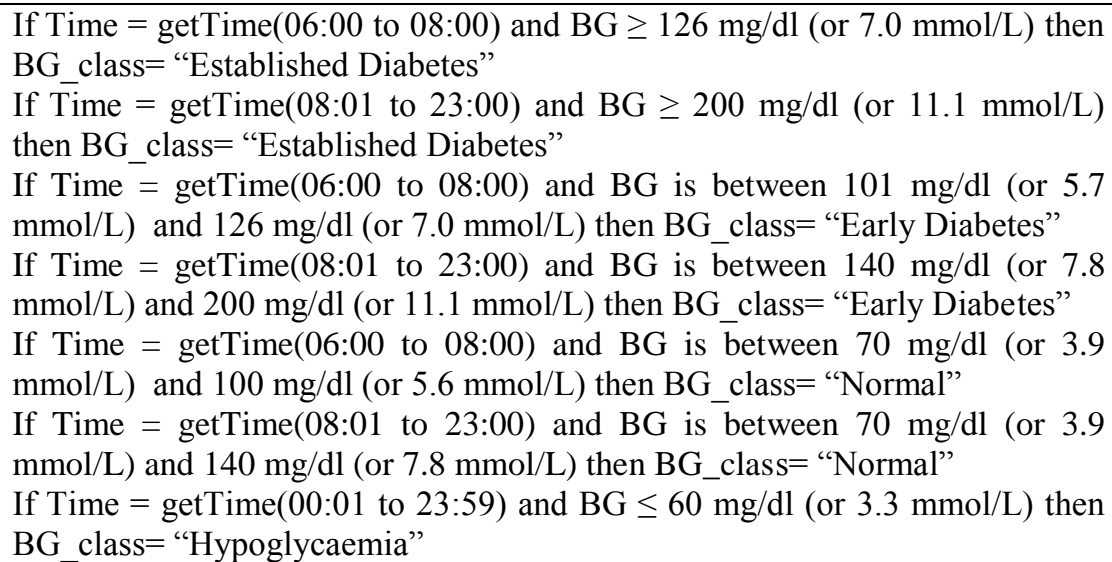 \\
\hline Blood Pressure (BP) & $\begin{array}{l}\text { If Systolic }<90(\mathrm{~mm} \mathrm{Hg}) \text { or Diastolic }<60(\mathrm{~mm} \mathrm{Hg}) \text { then } \mathrm{BP} \text { class }=\text { "Low" } \\
\text { If Systolic is } 90 \text { to } 119(\mathrm{~mm} \mathrm{Hg}) \text { and Diastolic is } 60 \text { to } 79(\mathrm{~mm} \mathrm{Hg}) \text { then } \\
\text { BP class }=\text { "Normal" } \\
\text { If Systolic is } 120 \text { to } 139(\mathrm{~mm} \mathrm{Hg}) \text { or Diastolic is } 80 \text { to } 89(\mathrm{~mm} \mathrm{Hg}) \text { then } \\
\text { BP class = "Pre-High" } \\
\text { If Systolic > } 140(\mathrm{~mm} \mathrm{Hg}) \text { or Diastolic }>90(\mathrm{~mm} \mathrm{Hg}) \text { then BP_class = } \\
\text { "High" }\end{array}$ \\
\hline Weight (WGT) & $\begin{array}{l}\text { If BMI }<18.5 \text { WGT_class }=\text { "Underweight" } \\
\text { If BMI }>18.5 \text { and BMI }<24.9 \text { WGT_class = "Normal" } \\
\text { If BMI }>25.0 \text { WGT class }=\text { "Overweight" }\end{array}$ \\
\hline
\end{tabular}




\begin{tabular}{|c|c|}
\hline $\begin{array}{l}\text { Heart Rate (HR) } \\
\text { (the cur_hr is the average } \\
\text { of heart rate during the } \\
\text { last one hour) } \\
\text { max_hr }=208-(\text { Age - } \\
\text { 0.7) }\end{array}$ & 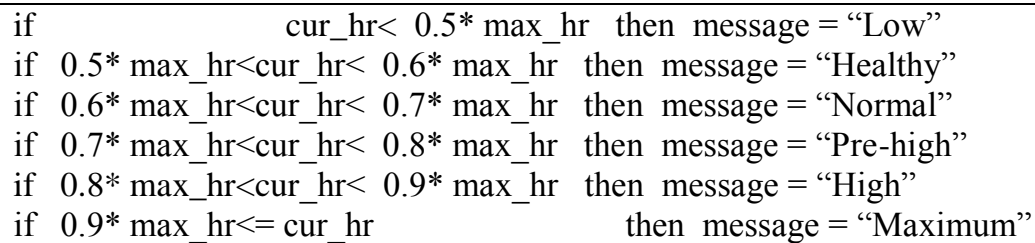 \\
\hline $\begin{array}{l}\text { Activity (the stps_cnt is } \\
\text { counted steps during last } \\
\text { one hour.) }\end{array}$ & 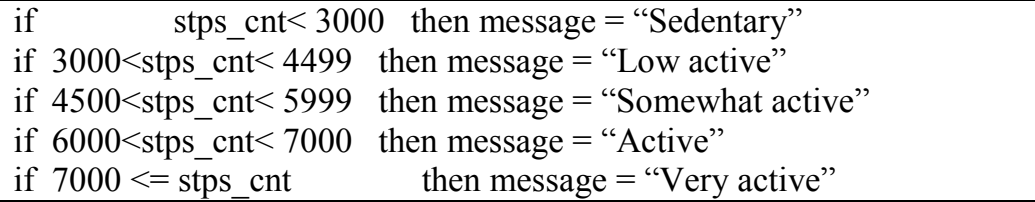 \\
\hline
\end{tabular}

Each health parameters are used as features and classes from the classified scheme are used as a feature value to create a new problem case. Again, a number of non-sensor features are extracted form the user related information, i.e. number of medications, number of comorbidity, score of selfassessment etc. Finally, the extracted features from sensor signal and user related information is combined together to create a new problem case and transferred into Step5.Step5 is the case-based

reasoning (CBR) classification scheme, which identifies the overall health condition of a user whether they are 'Healthy', 'More or Less Healthy' or 'Need Further Diagnosis'. Here, the retrieval function retrieves most similar cases by computing similarity value of two cases (i.e. new problem case and one solved case from case-library). An example of the case-library is show in Table 2, where a new case is being matched with Cases 1, 2 and others from the case-library. Table 2.An example of case-library representation

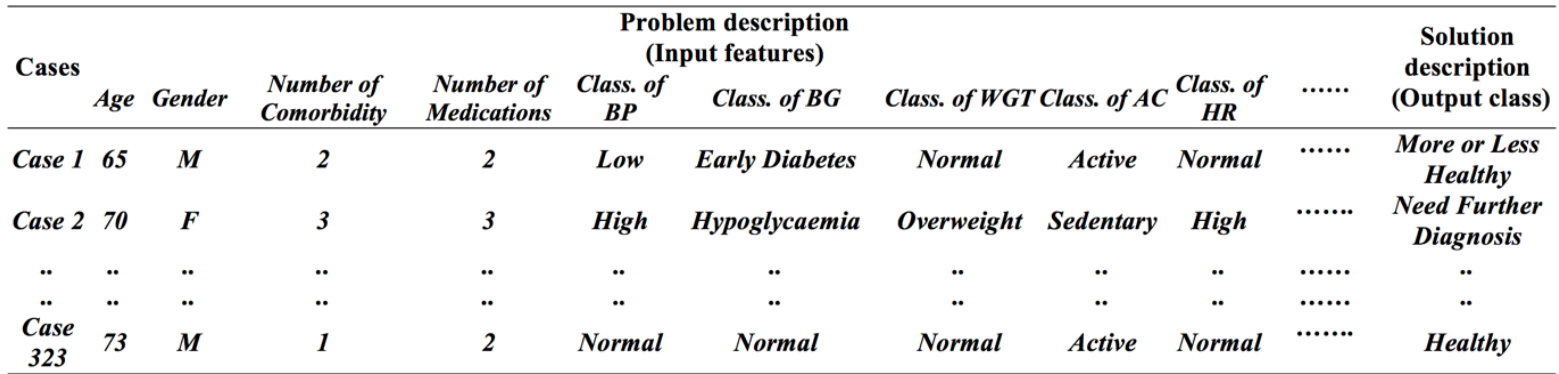

Weighting of features is an important task for retrieving similar cases. To determine the degree of importance of each feature, expert knowledge is needed. In this work, a domain expert defines weights of the features and weights are then used to retrieve similar cases. Similarity of a feature value between two cases (i.e. a target case and one case from library) is measured using the normalized Manhattan distance between the feature values of the two cases. Non-numeric features such as gender is converted to a numeric value by substituting the contextual value with a numeric one ( 1 for male, 0 for female). The Manhattan distance function to calculate the similarity of a feature between two cases is shown in equation 1 , where $T_{i}$ and $S_{i}$ are the $i$-th feature values of target and source case, respectively.

$\operatorname{sim}\left(\mathrm{T}_{\mathrm{i}}, \mathrm{S}_{\mathrm{i}}\right)=1 \frac{\left|T_{i} \quad S_{i}\right|}{\max \left\{T_{i}, \operatorname{Max}(i)\right\} \min \left\{T_{i}, \operatorname{Min}(i)\right\}}$

Here, Max(i) and Min(i) represents the Maximum and Minimum value of the feature $\mathrm{i}$ obtained from the whole case library. Then "max" and "min" functions compare the values between the new case feature $\mathrm{T}_{\mathrm{i}}$ and Maximum and Minimum values obtained from the case library. The function returns 1 if the values are the same and returns 0 if the values are dissimilar. This is known as a local similarity function.

$\operatorname{sim}(T, S)=\frac{\sum_{i}^{n} w_{i} \times \operatorname{sim}\left(T_{i}, S_{i}\right)}{\sum_{i}^{n} w_{i}}$

The similarity between two cases is then measured using the weighted average of all the features that are to be considered. The function for calculating similarity between two cases $T$ and $S$ with $n$ features is presented in equation 2 , where $\mathrm{w}_{\mathrm{i}}$ is the weight of the feature identified by an expert of the domain.

Finally, Step6 the outcome of the proposed system, where the personalized feedbacks, recommendations and alarms are generated based on the overall health condition identified by the CBR classification scheme.

\section{IV.EXPERIMENTAL WORKS}

The experimental work is conducted in three folds, 1) proof-of-concept prototype2) classification accuracy of the CBR retrieval, and 
3)a user evaluation.

\subsection{The proof-of-concept prototype}

In order to provide an intelligent health-IoT framework to monitor vital signs in daily life some components are implemented, such as: 1) android based user-interface and collector 2) gateway/switch server and 3)remote intelligent health server. The health related sensor devices are run by battery and communicate via Bluetooth communication. The sensor devices are paired with a Tablet where android operating system is installed. In the Tablet, user-interface and collector are running, where the collector receives health measurements using Bluetooth communication from the sensor devices upon user request through the user-interface. A screen short of the user interface is sown in figure 4.

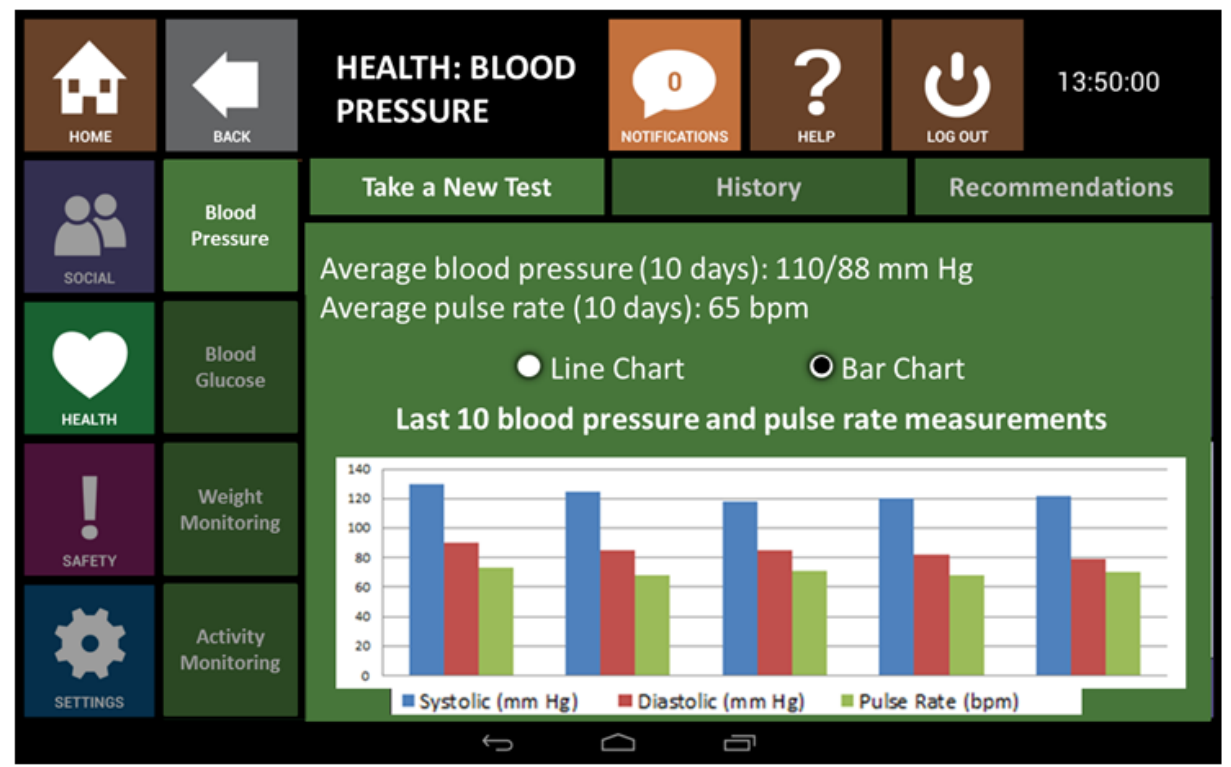

Figure 4. User interface showing last 10 days Blood pressure report

The Collector is based on native Java source code, which is in general the main programming language for Android devices. As soon as the collector receives the measurements it transfers them to the intelligent health gateway through https protocol and SOAP web service communication. The measurements are sent as a zip file including timestamps, battery and some other related information. The user interface is developed using JAVA programming language so that it can run in Android 4.0 operating system considering the Google Nexus 10 device. The gateway/switch server mainly conveys the message among the collector, gateway/switch server and remote intelligent health server. Moreover, SessionID and UUID are for each user is generated. The remote intelligent health server stores all the raw measurements and generates message id for the communication. It also calculates daily and weekly historical summary and store them in a MySQL database. The user has a direct access to the HIS to see the historical summary through the userinterface. HIS also generates recommendation in weekly basis based on historical summary and raw data measurements. An example of some weekly recommendations are shown through the user interface is presented in figure 5 . 


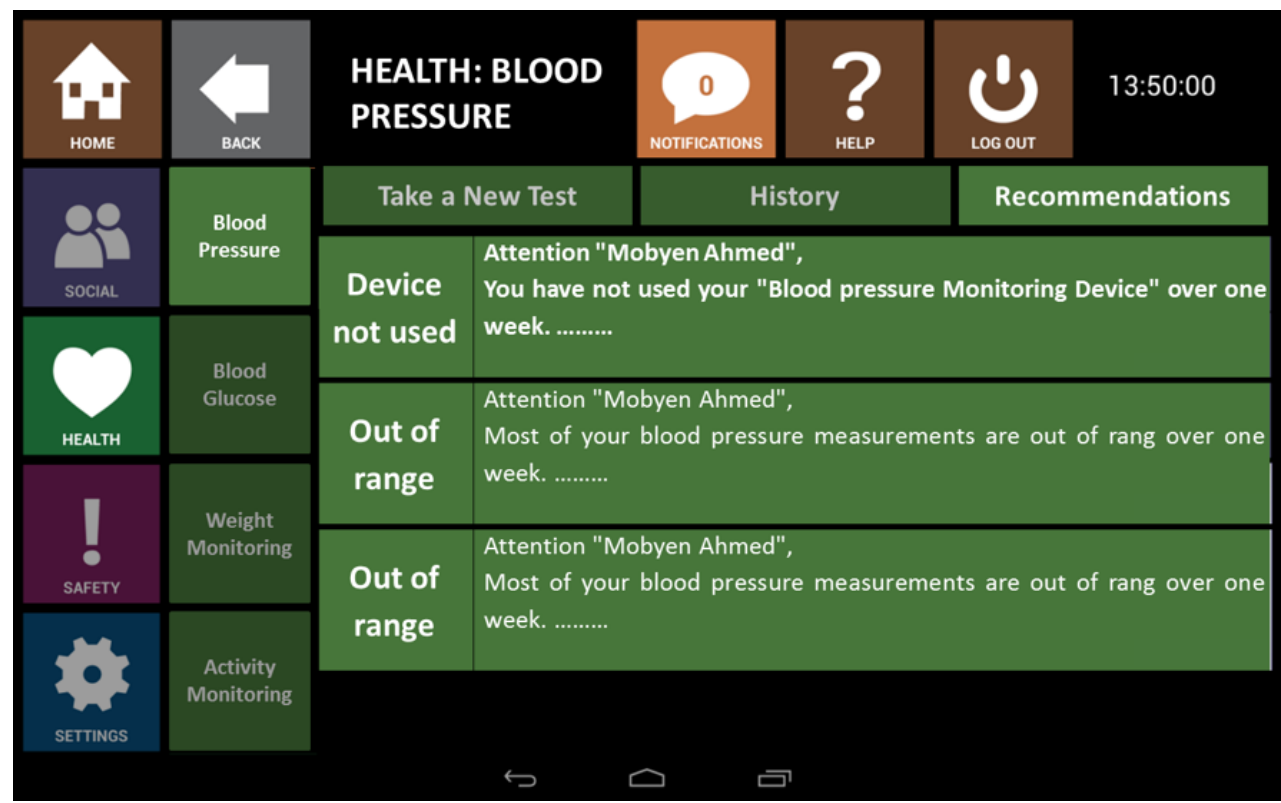

Figure 5. User interface showing weekly recommendations.

The web-based CDSS has been built using PHP and JavaScript programming language and case-libraries are developed in MySQL database. The main goal of this experiment is to see whether the several functions implemented in the webbased system are working properly or not. The DSS using case-based retrieval is verified by implementation as a prototype where all the implemented methods are compared according to their outcome, that is, in terms of the technical point of view. According to Watson [18], these trials have been conducted through the following 4 tests: 1) retrieval accuracy 2) retrieval consistency 3 ) case duplication and 4) global test. For the test retrieval accuracy, a "leave-one-out" retrieval technique is used i.e. one case is taken from the case library as a query case and then the system retrieves the most similar cases. Among the retrieved cases, the query case is also retrieved as the top similar case with the similarity value 1.0 i.e., the similarity value of two same cases is computed as $100 \%$. To test the retrieval consistency, the same query is used to perform more than one similar search and if it has been found that the same stored cases have been retrieved with the same similarity, then the implemented retrieval function is considered to have consistency. It is also observed that no cases are identical during retrieval except the query case, when it matches itself, thus case duplication is also checked. Regarding the global test, the classification accuracy of the CBR system is performed and discussed in the next section.

\subsection{Classification accuracy of CBR}

The classification accuracy of the CBR retrieval classification scheme has evaluated by developing a prototypical system where the main goal of the experiment is to see how accurately the CBR approach can classify. The case-library is built with 323 cases with 3 classes (i.e. 'Healthy', 'More or Less Healthy' and 'Need Further Diagnosis') in total. Here, for the retrieval, a "leave-one-out" retrieval technique is used i.e. one case is taken from the case library (i.e. 323 cases) as a query case and then the system retrieves the most similar cases. Among the retrieved cases, the top similar case is considered when i.e. $\mathrm{k}=1$, if the top case's class is matched with the query case class then we count the correct classification result as 1 . Similarly, top 2 similar cases are considered when i.e. $k=2$, if one of the 2 top most case's class is matched with the query case class then the classification is correct that denoted by 1 . The result of percentage of correctly classifications of each class is presented in Table 3 .

Table 3. Percentage of correctly classified cases for each class, where $\mathrm{k}=1$ means top most similar retrieved case, and $\mathrm{k}=2$ means top two similar retrieved cases are considered.

\begin{tabular}{llll}
\hline Classes & $\begin{array}{l}\text { Number of } \\
\text { Total Cases }\end{array}$ & $\begin{array}{l}\text { Percentage of Correctly Percentage of Correctly } \\
\text { Classified Cases }(\mathbf{k}=\mathbf{1})\end{array}$ & $\begin{array}{l}\text { Plassified Cases }(\mathbf{k}=\mathbf{2}) \\
\text { Clied }\end{array}$ \\
\hline Healthy & 195 & $\approx 76.9 \%(150)$ & $\approx 90.3 \%(176)$ \\
More or Less Healthy & 77 & $\approx 70.1 \%(54)$ & $\approx 87.0 \%(67)$ \\
Need Further Diagnosis & 51 & $\approx 78.4 \%(40)$ & $\approx 90.2 \%(46)$ \\
\hline
\end{tabular}




\begin{tabular}{lll}
\hline $\begin{array}{l}\text { Percentage of correctly } \\
\text { classified cases (in Average) }\end{array}$ & $\approx 75.1 \%$ & $\approx 89.2 \%$ \\
\hline
\end{tabular}

Table 4. Confusion matrix based on $\mathrm{CBR}$ classification considering $\mathrm{k}=1$

\begin{tabular}{llll}
\hline & Healthy & $\begin{array}{l}\text { More or } \\
\text { Less Healthy }\end{array}$ & $\begin{array}{l}\text { Need Further } \\
\text { Diagnosis }\end{array}$ \\
\hline $\begin{array}{l}\text { Healthy } \\
\begin{array}{l}\text { More or Less } \\
\text { Healthy }\end{array}\end{array}$ & $150(76.9 \%)$ & $37(19 \%)$ & $8(4.1 \%)$ \\
$\begin{array}{l}\text { Need Further } \\
\text { Diagnosis }\end{array}$ & $3(6 \%)$ & $54(70.1 \%)$ & $5(6.5 \%)$ \\
\hline
\end{tabular}

Table 5.Confusion matrix based on CBR classification considering $\mathrm{k}=2$.

\begin{tabular}{llll}
\hline & Healthy & $\begin{array}{l}\text { More or Less } \\
\text { Healthy }\end{array}$ & $\begin{array}{l}\text { Need Further } \\
\text { Diagnosis }\end{array}$ \\
\hline $\begin{array}{l}\text { Healthy } \\
\text { More or Less }\end{array}$ & $176(90.3 \%)$ & $15(7.7 \%)$ & $4(2.0 \%)$ \\
$\begin{array}{l}\text { Healthy } \\
\begin{array}{l}\text { Need Further } \\
\text { Diagnosis }\end{array}\end{array}$ & $7(9.1 \%)$ & $67(87.0 \%)$ & $3(3.9 \%)$ \\
\hline
\end{tabular}

As can be seen from Table 3, percentage of correctly classified cases by the proposed system considering $\mathrm{k}=1$ is 1 ) Healthy class $76.9 \%$ 2) More or Less Healthy class $70.1 \%$ and 3) Need Further Diagnosis class $78.4 \%$. Similarly, considering $\mathrm{k}=2$ is 1) Healthy class $90.3 \%$ 2) More or Less Healthy class $87.0 \%$ and 3) Need Further Diagnosis class $90.2 \%$. The confusion matrixes based on the proposed classification approach considering both $\mathrm{k}=1$ and $\mathrm{k}=2$ are presented in Table 4 and 5 . The distribution of the cases into three different classes can be observed from Table 4 where $\mathrm{k}=1$ and Table 5 where $\mathrm{k}=2$. Note that, considering $\mathrm{k}=2$ (Table 5), the cases belongs to the Healthy class are misclassified by $7.7 \%$ to the More or Less Healthy class and 2\% to the Need Further Diagnosis class. The cases belong to the More or Less Healthy class are misclassified by $9.1 \%$ to Healthy class and $3.9 \%$ to the Need Further Diagnosis class. Also, the cases belongs to the Need Further Diagnosis class are misclassified by $3.9 \%$ to the Healthy class and $5.9 \%$ to the More or Less Healthy class.

Table 6.Statistical Analysis of the system's classification

\begin{tabular}{lll}
\hline Criteria/Indices & Values $(\mathbf{k}=1)$ & Values $(\mathbf{k}=2)$ \\
\hline Total cases & 323 & 323 \\
Cases belong to Unhealthy group & 51 & 51 \\
(P) & & 272 \\
Cases belong to Healthy group $(\mathrm{N})$ & 272 & 46 \\
True positive (TP): & 40 & 7 \\
False positive (FP): & 13 & 265 \\
True negative (TN): & 259 & 5 \\
False negative (FN): & 11 & $\approx 0.90$ \\
Sensitivity = TP / (TP + FN) & $\approx 0.78$ & $\approx 0.97$ \\
Specificity = TN / (FP + TN) & $\approx 0.95$ & $\approx 0.96$ \\
Accuracy $=(\mathrm{TP}+\mathrm{TN}) /(\mathrm{P}+\mathrm{N})$ & $\approx 0.93$ & \\
\hline
\end{tabular}

A sensitivity, specificity and overall accuracy are also calculated and presented in Table 6, here, all the cases belongs to the More or Less Healthy and Healthy classes are treated as healthy group (e.g. N) and the cases belongs to the Need Further Diagnosis class are treated as Unhealthy group (e.g. P). According to Table 5, among the 323 cases, 272 cases are in Healthy group and 51 cases are Unhealthy group. After having True Positive (TP), False Positive (FP), True Negative (TN) and False
Negative (FN), the Sensitivity, Specificity and Accuracy were calculated both considering $\mathrm{k}=1$ and $\mathrm{k}=2$ criteria. The values obtained for $\mathrm{k}=1$ is Sensitivity $78 \%$, Specificity $95 \%$ and Accuracy 93\%; however, for $\mathrm{k}=2$ is Sensitivity $90 \%$, Specificity $97 \%$ and Accuracy $96 \%$.

\subsection{A user evaluation}

The composition of the trial formed by six participants from Spain $(n=3)$ and Slovenia $(n=3)$. 
They were invited to use the system at their own home at for eight weeks. After using the system for 8 weeks, user satisfactions were collected using a questionnaire (participants had to fulfil a questionnaire of satisfaction of the system) and the summary of this is presented in Table 7 and Table 8. The users satisfactions of Spanish participants for Navigation, Interface and both Performance are
3.3, 4.3, 3.6, and 3.6 in median (in Table 7), where Slovenia's participants the median score were 4.3 , 4.3, 2.2 and 3.3 for Navigation, Interface and both Performance (in Table 8). The median score on Overall quality of the System for Spanish participant was 7.3 and for Slovenia's participant was 6.6, however, the range was same for both the countries i.e. between 6 and 8 .

Table 7. User's $(\mathrm{N}=6)$ satisfaction using the system platform in Spain; VI=Very Inadequate, E=Excellent, SD=Strongly Disagree, D=Disagree, NAD=Neither Agree nor Disagree, A=Agree, SA=Strongly Agree, LQ=Low Quality, EQ=Excellent Quality.

\begin{tabular}{llll}
\hline No & $\mathbf{n}=\mathbf{3}$ & \multicolumn{2}{c}{ Median Range } \\
\hline 1. & Quality of Navigation $(1=\mathrm{VI}, 5=\mathrm{E})$ & 3.3 & $(3-4)$ \\
2. & Login to the Interface $(1=\mathrm{VI}, 5=\mathrm{E})$ & 4.3 & $(4-5)$ \\
3. & Performance in Health measures $(1=\mathrm{VI}, 5=\mathrm{E})$ & 3.6 & $(3-4)$ \\
4. & Performance in Requested old measurements $(1=\mathrm{VI}, 5=\mathrm{E})$ & 3.6 & $(3-4)$ \\
5. & Easy to use $(1=\mathrm{SD}, 2=\mathrm{D}, 3=\mathrm{NAD}, 4=\mathrm{A}, 5=\mathrm{SA})$ & 5 & $(5)$ \\
6. & Overall quality of the System $(1=\mathrm{LQ}, 10=\mathrm{EQ})$ & 7.3 & $(6-8)$
\end{tabular}

Table 8. User's $(\mathrm{N}=6)$ satisfaction using the system platform in Slovenia; VI=Very Inadequate, $\mathrm{E}=$ Excellent, $\mathrm{SD}=$ Strongly Disagree, $\mathrm{D}=$ Disagree, NAD=Neither Agree nor Disagree, $\mathrm{A}=\mathrm{Agree}$, SA=Strongly Agree, LQ=Low Quality, EQ=Excellent Quality.

\begin{tabular}{lllc}
\hline No & $\mathbf{n = 3}$ & \multicolumn{2}{c}{ Median Range } \\
\hline 1. & Quality of Navigation $(1=\mathrm{VI}, 5=\mathrm{E})$ & 4.3 & $(3-5)$ \\
2. & Login to the Interface $(1=\mathrm{VI}, 5=\mathrm{E})$ & 4.3 & $(3-5)$ \\
3. & Performance in Health measures (1= VI, 5=E) & 2.2 & $(2-5)$ \\
4. & Performance in Requested old measurements $(1=\mathrm{VI}, 5=\mathrm{E})$ & 3.3 & $(2-5)$ \\
5. & Easy to use (1=SD, 2=D, 3=NAD, 4=A, 5=SA) & 3.6 & $(3-5)$ \\
6. & Overall quality of the System (1=LQ, 10=EQ) & 6.6 & $(6-8)$ \\
\hline
\end{tabular}

\section{V.SUMMARY AND DISCUSSION}

Vital signs monitoring at home in daily life is becoming increasingly popular and promising solution and it is important for the primary users' especially for elderly care. This can be even more beneficial and interesting for the users within this area while it could work as a personalized selfserved system. This paper describes an intelligent health-IoT framework to monitor vital signs in daily life by combining general rules and casebased reasoning (CBR). Here, a set of general rules presented in Table 1 are used to classify individual health parametersand the CBR is applied to generate overall health condition of a user. The CBR uses the solutions of most similar cases retrieved by the proposed system compare to a new problem case. The user's contextual, medical, medicine, preference, self-assessment related information also assists the system to generate feedback, recommendation, reminder and alarm in a personalized manner.

Health related sensor readings from users are gathered remotely through mobile/tablets and other wireless devices; data are stores in the cloud and can be accessible from anywhere by anyone; the remote data were examined, analysed, and finally a decision has been taken by the DSS/ES or Doctors/clinicians; feedbacks, recommendations, reminders, and alarms are generated and provide to the user. The communications between the devices are done using Wi-fi/Bluetooth/Zigbee and HTTPS web services. Thus, to support all of these facilities in sensor data management a generic system-level framework for a self-served health monitoring system through the Internet of Things (IoT) is proposed and presented in [25],[26]. Here, the paper elaborates the overview of the system together with the system-level framework and its individual components.

A light version of the self-served health monitoring system is implemented by applying the proposed framework and approach. The evaluation is conducted in three steps:1) proof-of-concept prototype 2) classification accuracy of the CBR retrieval has observed where percentages of correctly classified cases, sensitivity, specificity and overall accuracy have calculated, and 3) a user evaluation. The proposed approach has evaluated considering six participants $($ Spain=3, Slovenia=3) where each participant has used the health monitoring system in their daily life for eight weeks. The main reason to use only six participants 
is to observe user's satisfaction based on a longer period of use (e.g. 8 weeks) in user's home and daily life environment without any supervision. Moreover, the project has had a limited number of sensors and tablets (e.g. only 6 kits) for the long time user evaluation. In Step2, the percentage of correctly classified cases have calculated both for $\mathrm{k}=1$ and $\mathrm{k}=2$. The reason behind choosing the number of retrieved cases as 2 (e.g. $\mathrm{k}=2$ ) is that the similarity value of two cases are very similar to each other, however, they are belonging to two different classes. The average value of the percentage of correctly classified classes, considering $\mathrm{k}=1$ is $75.1 \%$ and $\mathrm{k}=2$ is $89.2 \%$. The percentage of the classification increases while the system considers two top similar retrieved cases, here, the system also provides an alternative classification, which is closely similar, the new query case. Statistical analysis has performed to observed the performance of the proposed approach, and it has seen that overall system accuracy is $93 \%$ in CBR retrieval classification scheme considering $\mathrm{k}=1$ and it was $96 \%$ whole considering $\mathrm{k}=2$. Thus, the case-based retrieval classification scheme shows the possibility of identification of overall health status of elderly. In step3, a different result between the countries has been observed, this is not that they are different countries; there was a Bluetooth pairing and $\mathrm{Wi}-\mathrm{Fi}$ connection problems observed during the evaluation for the participant in Slovenia. Spanish (in Table 7) participants reported that the login to the Interface and the Performance were adequate and the Navigation was easy to use. They strongly agreed about the Easy to use of the platform, as well as they felt very comfortable using the system. Slovenian (in Table 8) participants reported that the Navigation and the Interface were good and the Performance was adequate. Regarding the Easy to use, most of them were neither agree nor disagree. However, one participant did not enjoy using the system since there was a problem in Bluetooth pairing. Nevertheless, Overall quality of the system was graded between 6 and 8 in both countries, which is expectable in the initial evaluation. Thus, the initial evaluation result demonstrates the competence and performance of the implemented system through the proposed framework. Thus, the system generates feedback, recommendation and alarm in a personalized manner based on elderly's medical information and health parameters. However, in the future the proposed approach will be evaluated in a larger scale i.e., involving more participants.

\section{ACKNOWLEDGEMENT}

The authors would like to acknowledge the projects named ESS-H: Embedded Sensor Systems for Health Research Profile, the distributed environments E-care@home are funded by Knowledge Foundation and SAAPHO: Secure Active Aging: Participation and Health for the Old (aal-2010-3-035) is funded by the Call AAL (Ambient Assisted Living) within the Call 3..

\section{REFERENCE}

[1]. Simonov M., ZichR. and Mazzitelli F., Personalized Healthcare Communication in Internet of Things, Proc. of URSI GA08, (2008).

[2]. Sidén J., Skerved V., Gao J., Forsström S., Nilsson H. E., Kanter T., and Gulliksson M., Home care with NFC sensors and a smart phone. In Proceedings of the 4th International Symposium on Applied Sciences in Biomedical and Communication Technologies (ISABEL '11). ACM, New York, NY, USA, (2011). Article 150, 5 pages. $\quad$ DOI=10.1145/2093698.2093848 http://doi.acm.org/10.1145/2093698.209384 8

[3]. Parra J., Hossain M.A, Uribarren A., Jacob E., RESTful Discovery and Eventing for Service Provisioning in Assisted Living Environments. Sensors. (2014); 14(5): $9227-$ 9246.

[4]. Ahmed M.U., Banaee H., Loutfi A., RafaelPalou X., Intelligent Healthcare Services to Support Health Monitoring of Elderly, In the International Conference on IoT Technologies for HealthCare, HealthyIoT, Rome, Italy, (2014).

[5]. Ahmed M.U., Espinosa J. R., Reissner A., Domingo À., Banaee H., Loutfi A., RafaelPalou X., Self-Serve ICT-based Health Monitoring to Support Active Ageing, in Conference $8^{\text {th }}$ International Conference on Health Informatics HEALTHINF, Lisbon, Portugal, (2015).

[6]. Armstrong S., Wireless connectivity for health and sports monitoring: A review. Br. J. Sports Med. (2007); 41:285-289.

[7]. Health@Home, Available online: http://www.aal-

europe.eu/projects/healthhome/(accessed on March,2017).

[8]. Geng Y., Li X., Mantysalo M., Xiaolin Z., Zhibo P., Li D. X., Kao-Walter S., Qiang C., Li-Rong Z., A Health-IoT Platform Based on the Integration of Intelligent Packaging, Unobtrusive Bio-Sensor, and Intelligent Medicine Box, Industrial Informatics, IEEE Transactions on, 10(4), pp.2180-2191, doi: 10.1109/TII.2014.2307795, (2014). 
[9]. Kulkarni A., Satheet S., Healthcare applications of the Internet of Things: A Review, International Journal of Computer Science and Information Technologies (IJCSIT), Vol. 5 (5), (2014), 6229-6232.

[10]. Salih A. S. M., Abraham A., A Review of Ambient Intelligence Assisted Healthcare Monitoring, International Journal of Computer Information Systems and Industrial Management Applications, Volume 5, pp.741-750, (2013).

[11]. Shyamal P., Hyung P., Paolo B., Leighton C., Mary R., A review of wearable sensors and systems with application in rehabilitation, Journal of NeuroEngineering and Rehabilitation, 9:21, (2012).

[12]. Strowig S.M., Raskin P., Improved glycemic control in intensively treated type 1 diabetic patients using blood glucose meters with storage capability and computer-assisted analysis. Journal of Diabetes Care, 21:1694 $-1698,(1998)$.

[13]. Understanding blood pressure readings. American Heart Association. http://www.heart.org/HEARTORG/Conditio ns/HighBloodPressure/AboutHighBloodPres sure/Understanding-Blood-PressureReadings_UCM_301764_Article.jsp, (accessed on March, 2017).

[14]. Ahmed M. U., Loutfi A., Physical Activity Identification using Supervised Machine Learning and based on Pulse Rate, Advanced Computer Science \& Application, 4, (2013).

[15]. Aamodt A., Plaza E., Case-based reasoning: Foundational issues, methodological variations, and system approaches, AI Communications, 39-59, (1994).

[16]. Watson I., Applying Case-Based Reasoning: Techniques for Enterprise systems, (1997).

[17]. Ahmed M.U., Begum S., Funk P., Xiong N., Schéele von. B., A Multi-Module Case Based Biofeedback System for Stress Treatment, international journal of Artificial Intelligence in Medicine, (2010).

[26]. MU Ahmed, M Björkman, M Lindén, A generic system-level framework for selfserve health monitoring system through internet of things (IoT), Studies in health technology and informatics 211, 305-307

[27]. Ahmed M.U., Funk P., A computer aided system for post-operative pain treatment combining knowledge discovery and casebased reasoning, International Conference on Case-Based Reasoning, 3-16
[18]. Begum S., Barua S., Ahmed M. U., Physiological Sensor Signals Classification for Healthcare using Sensor Data Fusion and Case-Based Reasoning, Sensor 14 (7), 11770-11785

[19]. Begum S., Ahmed M. U., Funk P., Xiong N., Folke M., Case-Based Reasoning Systems in the Health Sciences: A Survey on Recent Trends and Developments, IEEE Transactions on Systems, Man, and Cybernetics--Part C: Applications and Reviews, (2010).

[20]. Bichindaritz I., Marling C., Case-based reasoning in the health sciences: What's next? Artificial Intelligence in Medicine, 36(2), 127-135, (2006).

[21]. Culhane K. M., O'Connor M., Lyons D., Lyons G. M.,Accelerometers in rehabilitation medicine for older adults, Academic Journal of Age Ageing, 34(6): 556-60, (2005)

[22]. Alberti K.G.M.M., Zimmet P.Z, Definition, diagnosis and classification of diabetes mellitus and its complications. Part 1: diagnosis and classification of diabetes mellitus. Provisional report of a WHO Consultation, Journal of Diabetic Medicine, 15(7), pp 539-553, (1998)

[23]. Deurenberg P., Weststrate J.A., Seidell J. C., Body mass index as a measure of body fatness: age- and sex-specific prediction formulas, Br J Nutr, 65(2):105-14, (1991).

[24]. Catalogue of Projects, Ambient Assisted Living Joint Programme (AAL JP), 2011, http://www.aal-europe.eu/wpcontent/uploads/2012/04/AALCatalogue_onl ineV4.pdf, (accessed on March, 2017).

[25]. Ahmed M.U., BjörkmanM.,CausevicA.,Fotouhi H., Lindén M., An Overview on the Internet of Things for Health Monitoring Systems, $2^{\text {nd }} E A I$ International Conference on IoT Technologies for HealthCare (HealthyIoT2015) 